\title{
Poly-GR dipeptide repeat polymers correlate with neurodegeneration and Clinicopathological subtypes in C9ORF72- related brain disease
}

\author{
Nobutaka Sakae, Kevin F. Bieniek, Yong-Jie Zhang, Kelly Ross, Tania F. Gendron, Melissa E. Murray,
} Rosa Rademakers, Leonard Petrucelli and Dennis W. Dickson *i(

\begin{abstract}
Frontotemporal lobar degeneration (FTLD) is heterogeneous in clinical presentation, neuropathological characteristics and genetics. An expanded GGGGCC hexanucleotide repeat in C9ORF72 is the most common genetic cause of both FTLD and motor neuron disease (MND). Dipeptide repeat polymers (DPR) are generated through repeat-associated non-ATG translation, and they aggregate in neuronal inclusions with a distribution distinct from that of TDP-43 pathology. Recent studies from animal and cell culture models suggest that DPR might be toxic, but that toxicity may differ for specific DPR. Arginine containing DPR (poly-GR and poly-PR) have the greatest toxicity and are less frequent than other DPR (poly-GP, poly-GA). A unique feature of arginine-containing DPR is their potential for post-translational modification by methyl-transferases, which produces methylarginine DPR. In this report, we explored the relationship of DPR and methylarginine to markers of neurodegeneration using quantitative digital microscopic methods in 40 patients with C9ORF72 mutations and one of three different clinicopathologic phenotypes, FTLD, FTLD-MND or MND. We find that density and distribution of poly-GR inclusions are different from poly-GA and poly-GP inclusions. We also demonstrate colocalization of poly-GR with asymmetrical dimethylarginine (aDMA) immunoreactivity in regions with neurodegeneration. Differences in aDMA were also noted by clinical phenotype. FTLD-MND had the highest burden of poly-GR pathology compared to FTLD and MND, while FTLD-MND had higher burden of aDMA than FTLD. The results suggest that poly-GR pathology is associated with toxicity and neurodegeneration. It remains to be determined if dimethylarginine modification of poly-GR could contribute to its toxicity.
\end{abstract}

Keywords: C9ORF72, Dipeptide repeat polymers (DPR), Poly-GR, Neurodegeneration, Dimethylarginine

\section{Introduction}

Frontotemporal lobar degeneration (FTLD) is heterogeneous in clinical presentation, neuropathological characteristics and underlying genetic causes. Clinically, FTLD manifests in changes in behavior, personality and language, but it can also be associated with motor neuron disease (MND), including amyotrophic lateral sclerosis (upper and lower motor neurons affected), progressive muscular atrophy (lower motor neurons predominantly affected) and primary lateral sclerosis (upper motor neurons primarily affected) $[8,12,13]$. Hexanucleotide repeat expansions in

\footnotetext{
* Correspondence: dickson.dennis@mayo.edu

Department of Neuroscience, Mayo Clinic, 4500 San Pablo Road, Jacksonville, FL 32224, USA
}

(c) The Author(s). 2018 Open Access This article is distributed under the terms of the Creative Commons Attribution 4.0 International License (http://creativecommons.org/licenses/by/4.0/), which permits unrestricted use, distribution, and

chromosome 9 open reading frame 72 gene (C9ORF72) are the most common genetic cause of FTLD and MND [7, 24], which are collectively referred to as C9FTLD-MND. The neuropathological features of c9FTLD-MND include nuclear RNA foci, inclusions composed of repeat-associated non-ATG (RAN) translated dipeptide repeat polymers (DPR) and TDP-43 pathology. Of these features, DPR pathology and nuclear RNA foci are unique and highly specific to C9FTLD-MND. RAN translation can occur in both sense and antisense directions generating five different DPR: poly-(glycine-alanine/poly-GA), poly-(glycine-arginine/polyGR) and poly-(glycine-proline/poly-GP) encoded by the sense strand, and poly-(proline-alanine/poly-PA), poly-(proline-arginine/poly-PR) and poly-(proline-glycine/poly-PG) 
by the antisense strand $[1,11,21]$. In human brains, DPR inclusions are detected predominantly in neurons as cytoplasmic inclusions, but nuclear inclusions and rare inclusions in glial cells are also found [26]. There is evidence that DPR inclusions are detected in presymptomatic individuals before significant neurodegeneration and TDP-43 pathology is detected $[19,32]$. Recent progress in developing animal models of C9FTLD-MND suggests that toxicity of specific DPR polymers is variable. Several pathogenic mechanisms, not mutually exclusive, may be at play including nuclear dysfunction, altered RNA splicing, impaired nucleocytoplasmic transport, altered RNA granule dynamics, and disruption of proteostasis. Of the various DPR species, poly-PR and poly-GR are most toxic in Drosophila [20] and in cell culture models $[9,15,30,31,33,34]$. The reasons that poly-PR and poly-GR are more toxic remain unknown, but given their high arginine content, one might hypothesize that methylarginine post-translational modification might contribute to their toxicity. Arginine residues in polypeptides can be modified by methyltransferases to conjugate one (monomethylarginine) or two (dimethylarginine) methyl groups. Dimethylarginine modifications have been reported in proteins in human plasma and urine, and their levels are increased in conditions associated with enhanced protein breakdown, such as tumor growth and neurodegenerative disorders [27]. There are two isomers of dimethylarginine, symmetric dimethylarginine (sDMA) and asymmetric dimethylarginine (aDMA). The biologic function of DMA is not well known; however, elevated levels of aDMA in plasma predict poor prognosis in many diseases, such as cerebrovascular disease and Crohn's disease, where DMA modification is considered toxic [29]. The presence of DMA modifications has not been specifically studied in c9FTLD-MND.

Several studies have reported clinicopathological correlates of DPR in brains of c9FTLD-MND, but most have been relatively small autopsy series and used mostly semiquantitative methods $[6,17,26]$. It remains to be determined if there are correlations of specific DPR with clinical or neuropathological subtypes of C9ORF72-related disease. To address this issue, we sought evidence to support our hypothesis that arginine-containing DPR, poly-GR in particular, might correlate with the severity of neuropathology and that DMA modification might be related to a gain of toxicity in poly-GR. To investigate this, we systematically evaluated sense strand DPR (poly-GA, poly-GP and poly-GR), as well as aDMA in 40 patients with FTLD, FTLD-MND or MND. We found that poly-GR pathology correlated with neurodegeneration and clinicopathologic subtype. Further, we detected a correlation between the distribution of poly-GR and aDMA pathologies. Taken together, our results suggest a possible mechanism of poly-GR toxicity that could be the basis of novel therapeutic approaches.

\section{Material and methods Case materials}

Forty cases of FTLD or MND with C9ORF72 repeat expansion mutation were obtained from the Mayo Clinic brain bank. The C9ORF72 expansion carriers had pathological diagnoses of FTLD, MND or FTLD-MND. All cases were submitted to or autopsied by the brain bank for neurodegenerative disorders at the Mayo Clinic in Jacksonville, Florida. Clinical information (age at death, sex, clinical diagnosis, disease duration, and family history) was obtained from available medical records. The left hemibrain was fixed in $10 \%$ formalin, and the right hemibrain was frozen at $-80{ }^{\circ} \mathrm{C}$. Formalin-fixed tissue was sampled with standardized dissection methods and embedded in paraffin blocks.

\section{Genetic analyses}

All cases had hexanucleotide repeat expansions in C9ORF72 based on a repeat-primed polymerase chain reaction method that detects expansions of the GGGG $\mathrm{CC}$ hexanucleotide, and for most cases, the expansions was confirmed with Southern blotting. [7]

\section{Diagnostic neuropathology}

Gross and macroscopic neuropathological assessment was performed by standardized procedures. Formalin-fixed, paraffin-embedded tissue samples were cut at $5 \mu \mathrm{m}$ thickness and mounted on glass slides for further study. The neuropathologic assessment included immunohistochemistry for phospho-TDP-43 (pTDP-43; mouse monoclonal; Cosmo Bio USA, Carlsbad, CA) and C9RANT antibody [1]. A TDP-43 pathologic subtype was assigned to each case using the harmonized classification scheme [18] based upon morphology and distribution of lesions in cortical and subcortical regions [14].

\section{Immunohistochemistry and double-label immunofluorescence}

Immunohistochemistry was performed on $5-\mu \mathrm{m}$ thick sections from formalin-fixed paraffin embedded tissue. Glass-mounted sections were de-parafinized in xylene and rehydrated in ethanol and $\mathrm{dH}_{2} \mathrm{O}$. Rabbit polyclonal antibodies used for DPR immunohistochemistry were as follows: poly-GA (Rb9880), poly-GP (Rb5823), poly-GR (Rb7810) (Leonard Petrucelli, Mayo Clinic, Jacksonville, FL $[1,11]$ ). A rabbit polyclonal antibody (ASYM24 07414, MilliporeSigma, Burlington, MA) was used for immunohistochemistry for aDMA. Regions of interest were middle frontal gyrus (FCtx), motor cortex (MCtx) and hippocampus. Adjacent sections were immunostained for phospho-TDP-43 as above. All Immunoperoxidase sections were processed on a DAKO AutostainerPlus (Agilent/DAKO, Santa Clara, CA) with the DAKO EnVisionTM+ system-HRP with diaminobenzidine as the 
chromogen. Nonspecific antibody binding was blocked with normal goat serum (Sigma, St Louis, MO).

For double immunofluorescence staining, a rat monoclonal antibody was used to detect poly-GR (5H9, MilliporeSigma, Burlington, MA) and a rabbit polyclonal antibody was used to detect aDMA (MilliporeSigma, Burlington, MA). The fluorochromes were Alexa Fluor 568 and Alexa Fluor 488 conjugated to anti-rabbit or anti-rat IgG (Invitrogen/ThermoFisher, Waltham, MA).

\section{Assessment of neurodegeneration}

For assessment of neurodegeneration, H\&E stained sections of FCtx, MCtx and hippocampus were evaluated. Degeneration was graded semiquantitatively as absent (0), mild (1), moderate (2) or severe (3) based on the presence of spongiosis, neuronal loss and gliosis. In FCtx, neurodegeneration was typically associated with superficial laminar spongiosis, while in the hippocampal dentate fascia, discontinuity in the density of granular neurons was used as an indicator of neuronal loss and neurodegeneration. In hippocampal subfields - CA4 and CA2/3 - degeneration was assessed by neuronal loss and astrogliosis (see Additional file 1: Figure S1).

\section{Image analysis}

Digital microscopy methods used in this study have been described previously [22]. Briefly, immunostained glass slides were scanned on an Aperio ScanScope XT slide scanner (Aperio Technologies/Leica Biosystems, Buffalo Grove, IL) producing high resolution digital images. The regions of interest in the hippocampus included the entire hippocampus, as well as delimited subregions of dentate fascia, CA4 sector and CA2/3 sector. In MCtx and FCtx, the region of interest was the entire thickness of the cortex where the pial surface was parallel to the cortical gray-white junction. Digital image analysis was performed using Aperio ImageScope software. A color deconvolution algorithm was used to count the number of pixels that were strongly immunostained by the chromogen. The output variable was percentage of strong positive staining relative to total pixels in the region of interest.

Analytic algorithms for poly-GA had to be adapted to exclude signals two standard deviations above and below the mean because some cases had unexplained background staining. Given this limitation, we also used manual counting methods for poly-GA, as well as for aDMA. In all cases, aDMA labeled not only cytoplasmic inclusions, but also many nuclei presumably due to detection of methylated histones or other nuclear proteins [10]. The poly-GA and aDMA manual counts were performed on digital images. In order to make comparisons between the various DPR, it was also necessary to perform manual counts for poly-GP and poly-GR. The number of inclusions detected from manual counts was assessed in the same area traced for image analysis. Manual counts and positive pixel burden from color deconvolution image analysis were highly correlated for both poly-GP and poly-GR (Additional file 2: Figure S2). Only the motor cortex had poor correlation between poly-GP manual counts and positive pixel burden, in part due to prominent cytoplasmic immunoreactivity in large neurons, including Betz cells. These did not represent inclusions in that they had diffuse cytoplasmic staining, instead of compact cytoplasmic inclusion bodies, which were manually counted. As with image analysis, manual counts were expressed as density per square millimeter by dividing the manual count by total area of the annotated region of interest.

\section{Cell culture experiments}

HEK-293 T cells were grown in Opti-Mem plus 10\% FBS and $1 \%$ penicillin-streptomycin. To examine methylation of poly-GR proteins, HEK-293 T cells were grown in 12-well plates or on coverslips in 24-well plates. Cultures were transfected with $1 \mu \mathrm{g}$ (12-well plates) or $0.5 \mu \mathrm{g}$ (24-well plates) of an expression vector[GFP, GFP-(GR) 50 or GFP-(GR) ${ }_{100}$ using Lipofectamine 2000 (Life Technology). Four hours after transfection, the cultured cells were treated with methylarginine transferase inhibitor, AdOx (A7154, Sigma), at concentration of 5 or $20 \mu \mathrm{M}$. DMSO was used as vehicle control. Twenty-four hours after treatment, the cultured cells were harvested for Western blot analysis or fixed for immunofluorescence staining.

\section{Immunofluorescence staining}

HEK-293 T cells were fixed with 4\% paraformaldehyde in $\mathrm{PBS}$ for $15 \mathrm{~min}$, and then permeabilized with PBS-0.5\% Triton X-100 for $10 \mathrm{~min}$. To examine aDMA, cells were blocked with $5 \%$ nonfat milk for $1 \mathrm{~h}$ at room temperature, and then incubated overnight at $4{ }^{\circ} \mathrm{C}$ with rabbit polyclonal anti-aDMA antibody (ASYM24, Millipore). After washing, cells were incubated with the corresponding Alexa Fluor 568-conjugated goat anti-rabbit secondary antibody (1:500, Molecular Probes) at room temperature for $2 \mathrm{~h}$. After washing, they were mounted with Vectashield Mounting Media with DAPI (Vector Laboratories). Images were obtained on a Zeiss LSM 510 META confocal microscope. To quantify the number of cells positive for GFP and aDMA, microscopic fields were randomly selected at $40 \mathrm{X}$ magnification. For each field, the number of GFP-positive cells and aDMA-positive aggregates, as well as aggregates with double staining, were manually counted, blinded to condition. These counts were used to calculate the average percentage of aDMA aggregates in cells expressing GFP, GFP-(GR) $)_{50}$ or GFP-(GR) ${ }_{100}$. 
Biochemical studies of cell lysates

Cell pellets were lysed in co-immunoprecipitation (co-IP) buffer (50 mM Tris- $\mathrm{HCl}, \mathrm{pH} 7.4,300 \mathrm{mM} \mathrm{NaCl}$, 1\% Triton X-100, 5 mM EDTA) plus 2\% SDS, and both protease and phosphatase inhibitors, sonicated on ice, and then centrifuged at $16,000 \times \mathrm{g}$ for $20 \mathrm{~min}$. Supernatants were saved as cell lysates. The protein concentration of supernatants was determined by BCA assay (Thermo Scientific) prior to Western blot analysis. Cell lysates were diluted with $2 \times$ SDS-loading buffer at a 1:1 ratio $(v / \mathrm{v})$, and then heated at $95{ }^{\circ} \mathrm{C}$ for $5 \mathrm{~min}$. Equal amounts of protein were loaded into 12-well 4-20\% Tris-glycine gels (Novex). After transferring proteins to membranes, membranes were blocked with 5\% nonfat dry milk in TBS plus $0.1 \%$ Tween 20 (TBST) for $1 \mathrm{~h}$, and then incubated with rabbit polyclonal anti-GFP antibody (A-6455, 1:4000, Life Technologies), rabbit polyclonal anti-GR antibody (Rb7810, 1:2000), rabbit polyclonal anti-aDMA antibody (07-414, 1:1000, EMD Millipore), or mouse monoclonal or GAPDH antibody (H86504M, 1:10000, Meridian Life Science) overnight at $4{ }^{\circ} \mathrm{C}$. Membranes were washed in TBST and incubated with donkey anti-rabbit or anti-mouse IgG antibodies conjugated to horseradish peroxidase (1:5000; Jackson ImmunoResearch) for $1 \mathrm{~h}$. Protein expression was visualized by enhanced chemiluminescence treatment and exposure to film.

\section{Statistical analyses}

Sigma Plot Version 12 (Systat Software, San Jose, CA) was used for statistical analyses. Due to small sample sizes, non-parametric Kruskal-Wallis analysis of variance on ranks (ANOVA on Ranks) was performed on quantitative measures to assess differences between the groups. Post hoc pairwise comparisons were performed between each of the groups using Mann-Whitney rank sum test. For categorical data (e.g., sex and APOE genotype), a Chi-square test was used to compare groups. Fisher's exact test was used for comparison of pairwise categorical data if the counts were less than 5. Correlative analysis was performed using Spearman rank order correlation. A $p$-value $<0.05$ was considered statistically significant.

\section{Results}

\section{Demographics features of study cohort}

The 40 patients with C9ORF72 mutations included 13 with FTLD, 14 with FTLD-MND and 13 with MND. Demographic information is summarized in Table 1. Patients with FTLD had significantly longer disease duration than patients with MND. They were also significantly older at death compared to both FTLD-MND and MND. The median age at death was 74 for FTLD, 61 for FTLD-MND and 56 for MND. Median disease durations were 6.4 years for FTLD, 3.6 years for FTLD-MND and 2.4 years for MND.
Table 1 Demographics of clinicopathologic subgroups

\begin{tabular}{llll}
\hline Subgroup & N (F/M) & Age at death & Disease duration \\
\hline FTLD & $13(2 / 11)$ & $74(71,83)^{*}$ & $6.4(4.4,10)^{*}$ \\
FTLD-MND & $14(7 / 7)$ & $61(60,68)^{*}$ & $3.6(2.2,5.5)$ \\
MND & $13(9 / 4)$ & $56(50,68)$ & $2.4(1.3,3.5)$
\end{tabular}

All variables analyzed with Kruskal-Wallis ANOVA on Ranks, and data are displayed as median (25th and 75th range), unless otherwise noted *Statistically significant $p$-value $(p<0.05)$; all $p$-values for ANOVA on Ranks comparison of all three groups

\section{Spectrum of poly-GA, poly-GP and poly-GR pathology}

In this study, we limited neuropathological analyses to sense strand DPR (poly-GA, poly-GP and poly-GR) given the paucity of inclusions and the lack of good detection reagents for antisense DPR (poly-PA and poly-PR). We focused particularly on poly-GR pathology, and compared the pathology of poly-GR with poly-GP and poly-GA pathology as these have been shown by both our previous studies and those of others to be the most abundant DPR species in brain samples. Consistent with previous reports, we found that most of the DPR inclusions were neuronal cytoplasmic inclusions (Fig. 1); dystrophic neurites were less frequent $[1,11,16,17,21,26]$. In contrast to poly-GP and poly-GA, we did not detect dystrophic neurites with immunohistochemistry for poly-GR. These results are consistent with a previous study that showed almost complete lack of poly-GR immunoreactive dystrophic neurites [26]. Glial cytoplasmic inclusions were also occasionally detected with poly-GA and poly-GP immunohistochemistry, but they were very rare with poly-GR immunohistochemistry.

\section{Poly-GR inclusion distribution among clinicopathologic subgroups}

To evaluate whether poly-GA, poly-GP or poly-GR pathology differs among different clinicopathologic subgroups, we quantified lesion burden with image analysis. Although there were no significant differences between the subgroups for burden of poly-GA and poly-GP, poly-GR was significantly different in the three clinicopathologic subgroups. While overall lesions were sparse, we found greater burden of poly-GR inclusions in the FCtx of FTLD-MND compared to MND, and significantly more poly-GR inclusions in specific hippocampal subfields (i.e. CA2/3) of FTLD-MND compared to FTLD, as well as a trend for increases in CA4 of FTLD-MND compared to FTLD (Additional file 3: Table $\mathrm{S} 1$ ). We found poly-GR density was significantly different in FTD/MND in frontal cortex and hippocampus.

\section{Correlations between poly-GR pathology and neurodegeneration}

We hypothesized that if poly-GR is neurotoxic, the density of poly-GR inclusions might be associated with 


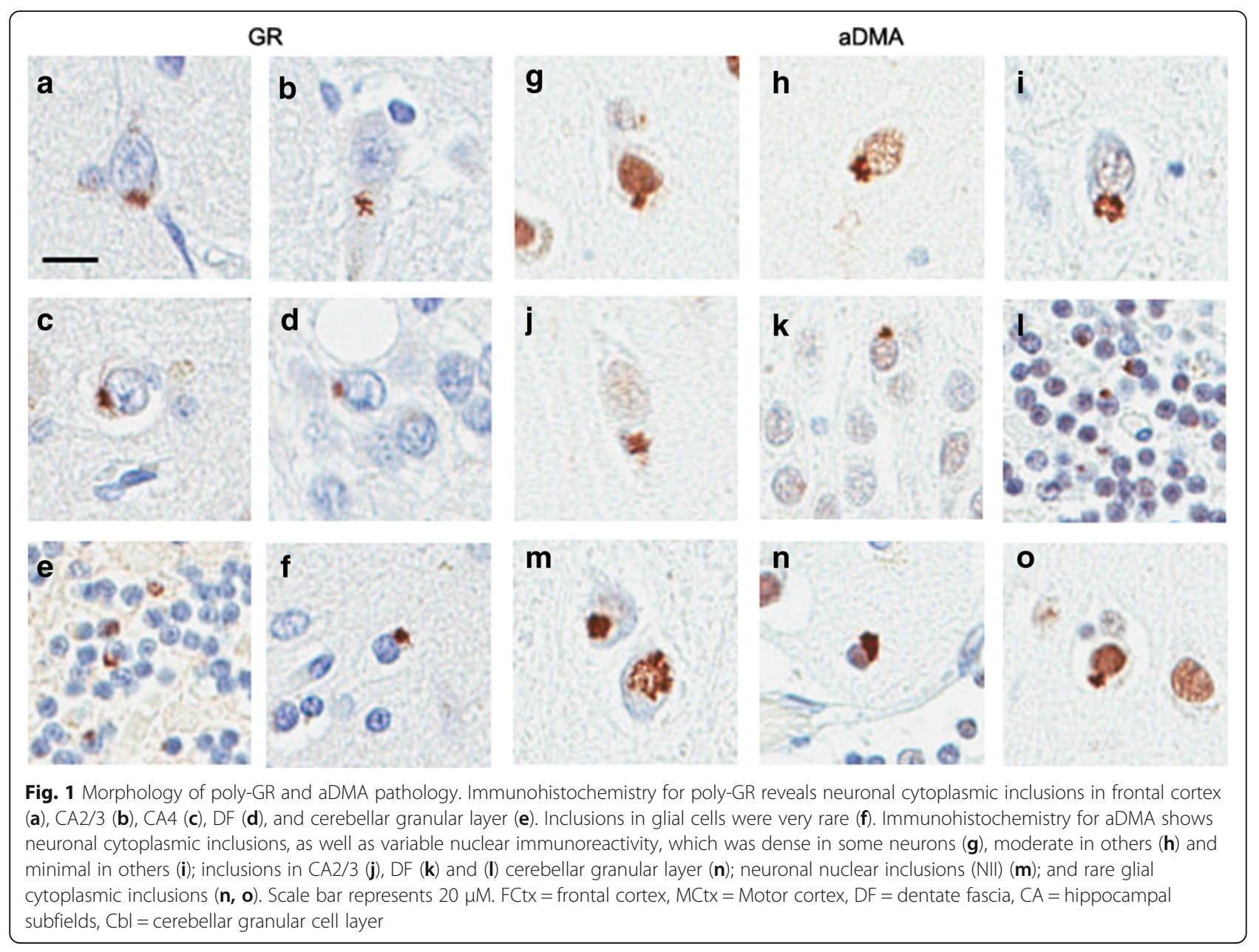

neurodegeneration. We thus analyzed the burden of poly-GA, poly-GP and poly-GR, as well as pTDP-43 using a color deconvolution algorithm of digital images of regions of interest. In the same regions we scored the severity of neurodegeneration with semiquantitative methods. As expected, there were strong correlations between density of pTDP-43 pathology and neurodegeneration in FCtx $(r=0.46, p=0.004)$, hippocampal DF $(r=0.40, p=0.01)$ and CA4 $(r=0.61$, $p=0.00006$ ) (see Additional file 4: Figure S3). Interestingly, we also found a strong correlation between poly-GR density and neurodegeneration in FCtx $(r=$ $0.45, p=0.004$ ), but a less robust correlation between poly-GR density and neurodegeneration in the hippocampal DF $(r=0.33, p=0.04)$ (Table 2). Representative images of poly-GR and neurodegeneration are illustrated in Fig. 2. In contrast to other sense-strand DPR (poly-GA and poly-GP), poly-GR showed correlation with neurodegeneration even though the density of lesions was far less for poly-GR compared with poly-GA and poly-GP.

\section{Spectrum and distribution of poly-GR pathology and aDMA distribution}

To assess our hypothesis that DMA modification may contribute to a possible toxic gain of function in poly-GR inclusions, we studied the morphology and distribution of inclusions immunoreactive for aDMA. We also quantified

Table 2 Correlations of phospho-TDP-43 and DPR burden with neurodegeneration

\begin{tabular}{llll}
\hline & FCtx & DF & CA4 \\
\hline poly-GA & $r=-0.19$ & $r=0.20$ & $r=0.10$ \\
& $p=0.28$ & $p=0.24$ & $p=0.57$ \\
poly-GP & $r=-0.14$ & $r=0.26$ & $r=0.31$ \\
& $p=0.42$ & $p=0.12$ & $p=0.06$ \\
poly-GR & $r=0.45$ & $r=0.33$ & $r=-0.01$ \\
& $p=0.004$ & $p=0.04$ & $p=0.96$ \\
pTDP-43 & $r=0.46$ & $r=0.40$ & $r=0.61$ \\
& $p=0.004$ & $p=0.013$ & $p=0.00006$ \\
\hline
\end{tabular}

$P$-values are from Pearson's test of correlation. Significant $p$-values $(<0.05)$ $F C t x$ frontal cortex, DF dentate fascia, CA4 cornu ammonis sector 4 


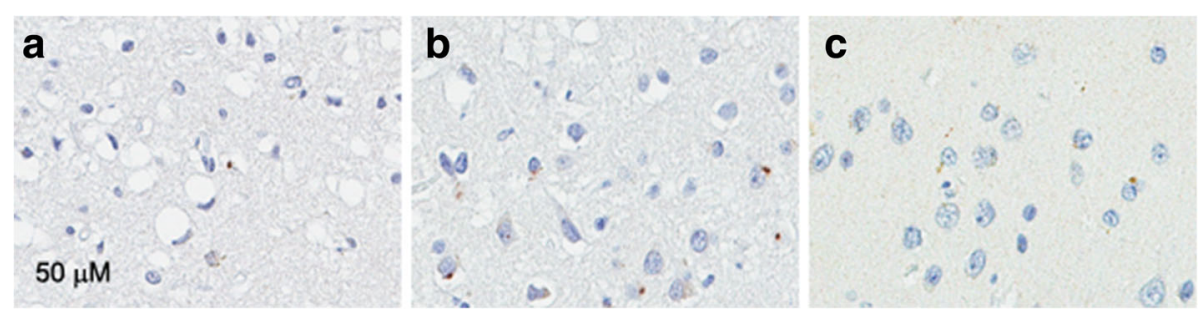

Fig. 2 poly-GR density and neurodegenerationpoly-GR density is associated with neurodegeneration in FCtx and DF. Representative images show neurodegeneration and poly-GR inclusions. Most severe neurodegeneration with spongiosis and gliosis, but sparse poly-GR inclusions in FTLD (a) Severe neurodegeneration with spongiosis and gliosis in FTLD-MND (b) Mild neurodegeneration in MND (c). Scale bar represents $50 \mu M$

aDMA using western blot analysis in a subset of cases included in the larger immunohistochemical study. Blots were also probed with antibody to sDMA. The focus of this sub-analysis was on four FTLD-MND patients that had the most significant poly-GR pathology. Four FTLD-MND patients without C9ORF72 mutations were included for comparison. We detected high molecular weight aDMA-immunoreactive species in brain lysates of c9FTLD-MND (Additional file 5: Figure S4), but not of sporadic FTLD-MND. We did not detect any differences between c9FTLD-MND and sporadic FTLD-MND for sDMA (Additional file 5: Figure S4).

Given these results, we focused only on aDMA in subsequent histopathologic studies. We characterized the spectrum of poly-GR and aDMA pathology in C9ORF72 patients. As mentioned above, poly-GR neuronal inclusions were observed throughout the brain. Only a few glial inclusions and isolated neuronal intranuclear inclusions were detected, while no dystrophic neurites were detected (Fig. 1). Immunohistochemistry for aDMA labeled neuronal inclusions as well as many nuclei, the latter probably related to physiologic methylation of nuclear proteins such as histones (Fig. 1). As we observed for poly-GR, most of the inclusions immunoreactive for aDMA were neuronal cytoplasmic inclusions; glial inclusions were sparse, and no dystrophic neurites were found.

\section{Similar distribution of poly-GR and aDMA immunoreactivities in C9FTLD-MND brains}

We used double immunofluorescence labeling to determine what proportion of the poly-GR inclusions also had aDMA immunoreactivity. We found frequent colocalization of poly-GR and aDMA in neuronal inclusions of the DF and CA4 sector of the hippocampus (Fig. 3). We performed manual counts of poly-GR and aDMA immunoreactive inclusions because excessive nuclear signal of aDMA precluded use of image analysis methods. We foun that manual counts and image analysis results were highly correlated (Additional file 2: Figure S2). We found similar densities and distributions of poly-GR and aDMA immunoreactive inclusions in FCtx, MCtx and hippocampal DF (Fig. 4a-c). As expected, there was a strong correlation between poly-GR and aDMA (Fig. 3) and this was true for all brain regions analyzed (Additional file 6: Table S2). Taken together, these data suggest that a significant portion of poly-GR pathology may be methylated in C9ORF72-related disease.

\section{Clinicopathological correlations of poly-GR and aDMA pathology based on manual inclusion counts}

We investigated poly-GA, poly-GP, poly-GR and aDMA immunoreactive inclusions in clinicopathologic subgroups, as assessed from manual counts. Similar to results described above, the density of poly-GR and aDMA immunoreactive neuronal inclusions was greater in patients with FTLD-MND compared to FTLD or MND. In the hippocampus, density of poly-GR inclusions was significantly greater in the DF, CA4 and CA2/3 of FTLD-MND compared to FTLD (Table 3). In addition, the density of aDMA inclusions was significantly greater in CA4 of FTLD-MND compared to FTLD. In frontal cortex, the density of poly-GR inclusions in FTLD-MND was significantly greater than in both FTLD and MND (Table 3). These results were similar to those obtained by image analysis using color deconvolution algorithms (Additional file 3: Table S1).

\section{In vitro evidence of poly-GR methylation}

To obtain further insight into poly-GR pathology in c9FTLD-MND, we overexpressed GFP-tagged poly-(GR) 50 or poly-(GR) ${ }_{100}$ and treated the cells with adenosine dialdehyde (AdOx), a global methyltransferase inhibitor, at two concentrations $(5 \mu \mathrm{M}$ and $20 \mu \mathrm{M})$. GFP-(GR $)_{50}$ accumulated only in the nucleus, whereas GFP-(GR) $)_{100}$ was detected in both the nucleus and cytoplasm. The cytoplasmic aggregates resembled inclusion bodies (Fig. 5). These results suggest that formation of poly-GR aggregates might be determined, in part, by repeat length, with longer repeats forming cytoplasmic inclusions resembling those seen in human brain cells (Fig. 1). To study the relationship between poly-GR aggregation and aDMA modification, we performed double immunofluorescent staining. There was frequent colocalization of poly-GR and aDMA in 

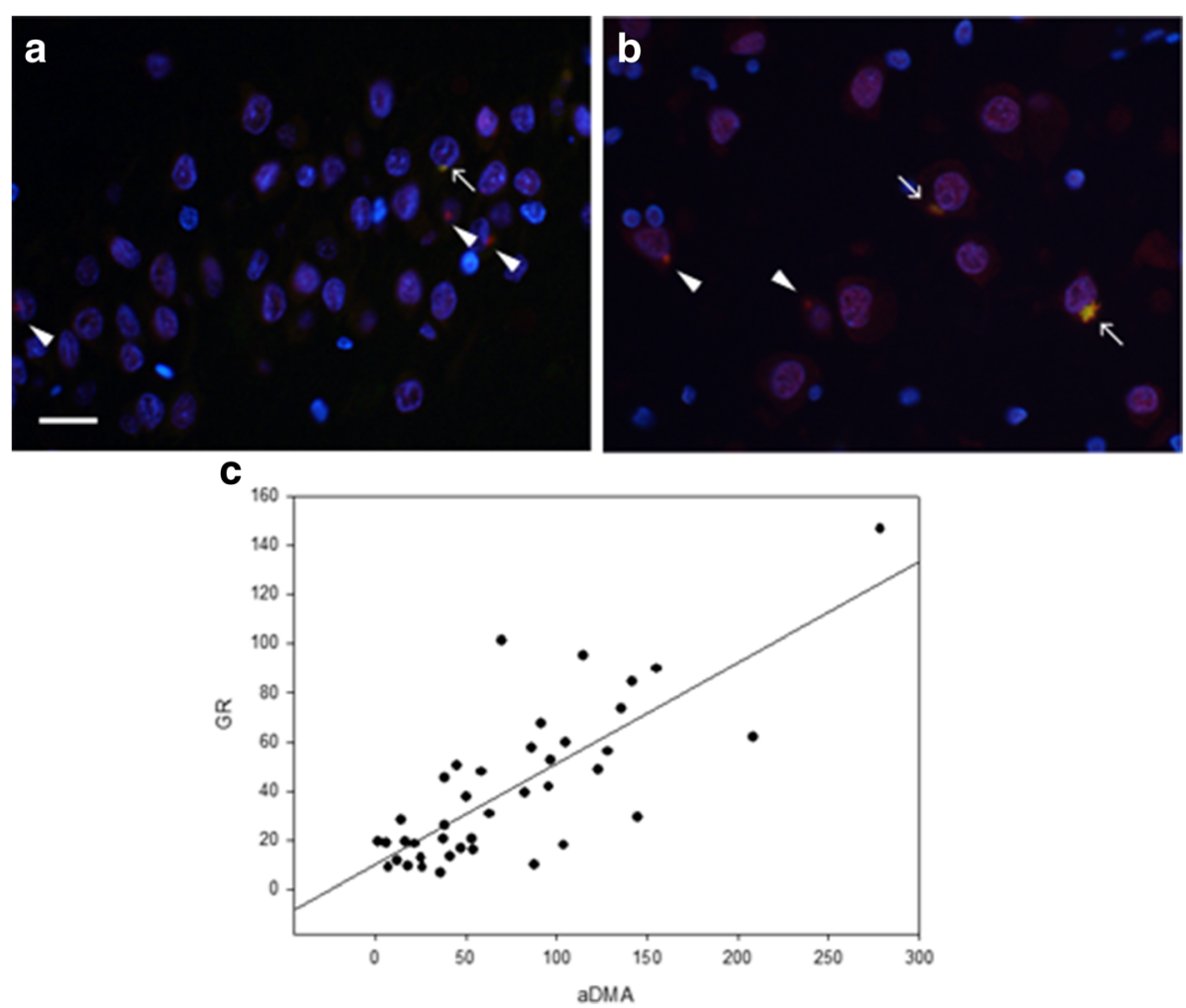

Fig. 3 Colocalization of poly-GR and aDMA. Poly-GR neuronal cytoplasmic inclusions in the dentate fascia and CA4 of the hippocampus. Sparse poly-GR inclusions in the dentate fascia show colocalization with aDMA (arrow). Note that not all poly-GR aggregates contain aDMA (arrowheads) (a). Moderate poly-GR inclusions show colocalization with aDMA in CA4 (arrows). Again, not all poly-GR aggregates contain aDMA (arrowheads) (b). Scale bars: 10 m. Plot shows the association of poly-GR and aDMA neuronal inclusions in the dentate fascia. The line shows linear regression $(r=0.77)(\mathbf{c})$
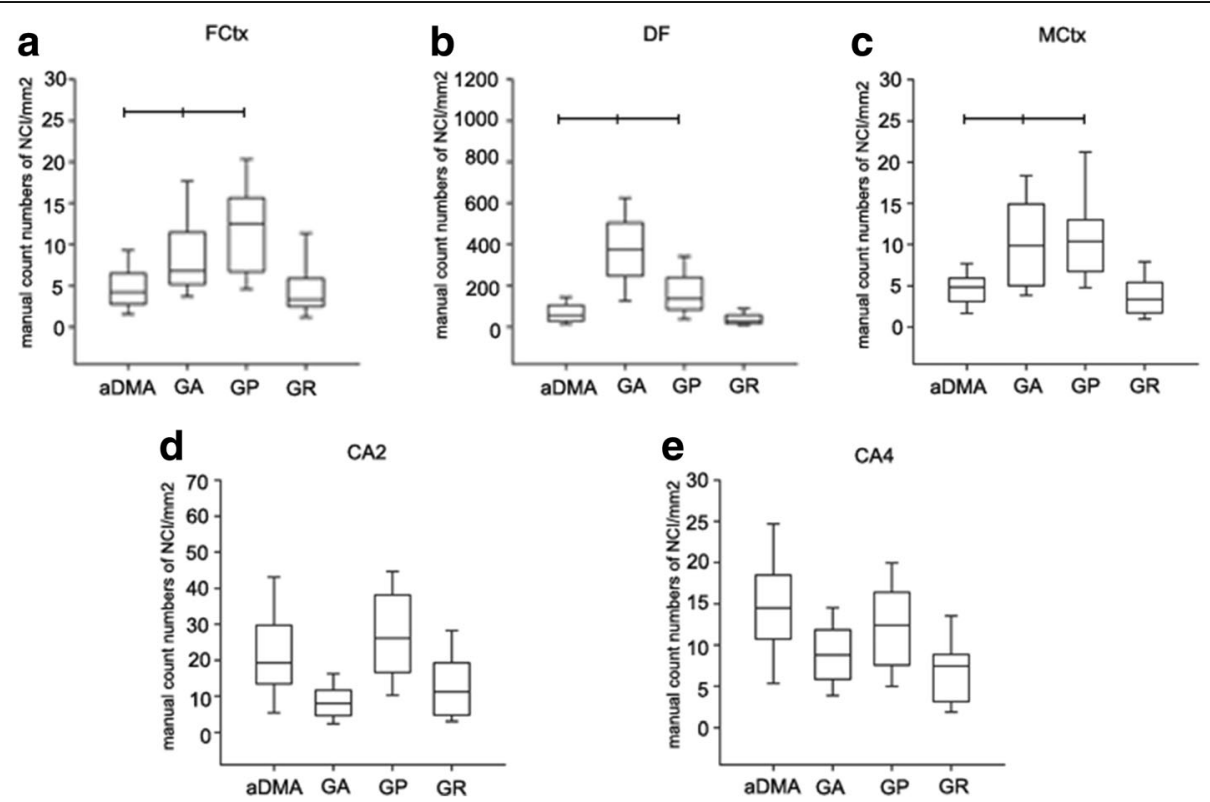

CA4

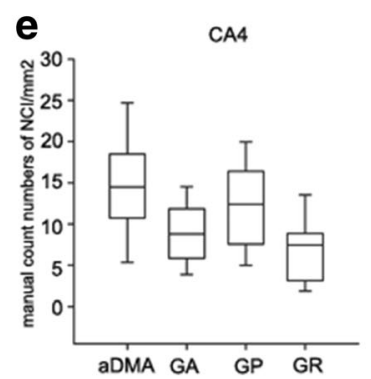

Fig. 4 Overall frequency of inclusions for sense strand DPR and aDMA. Quantitative analysis of poly-GA, poly-GR and aDMA density in frontal cortex, hippocampus and motor cortex. The total number of inclusions counted in each case and the density of inclusions were calculated by total numbers of inclusions/total stained area $\left(\mathrm{mm}^{2}\right)$. Frontal cortex (a), dentate fascia (b), motor cortex (c), CA2/3 (d), and CA4 (e). All variables analyzed with Kruskal-Wallis ANOVA on Ranks followed by Dunn's post hoc test and data are displayed as median (25th and 75th range). *Statistically significant $p$-value ( $p<0.05)$; all $p$-value for ANOVA on Ranks comparison of all groups 
Table 3 Burden of DPR and aDMA by region in clinicopathologic subgroups

\begin{tabular}{|c|c|c|c|c|c|}
\hline & FCtx & DF & CA4 & $\mathrm{CA} 2 / 3$ & MCtx \\
\hline & poly-GA & & & & \\
\hline FTLD & $6.4(3.8,9.4)$ & $310(110,470)$ & $6(4.1,9.5)$ & $5.6(4.5,8.3)$ & $5.8(3.7,12)$ \\
\hline FTLD-MND & $7.5(5.6,15)$ & $420(320,630)$ & $9.5(6.6,13)$ & $9.7(4.7,15)$ & $14(6.2,17)$ \\
\hline \multirow[t]{2}{*}{ MND } & $7.4(5.8,13)$ & $380(280,480)$ & $10(7.4,14)$ & $8.2(3.0,12)$ & $9.3(6.5,13)$ \\
\hline & poly-GP & & & & \\
\hline FTLD & $9.7(5.9,14)$ & $120(71,262)$ & $8(6.7,13)$ & $19(13,33)$ & $10(6.7,13)$ \\
\hline FTLD-MND & $13(11,18)$ & $180(98,270)$ & $13(7.4,18)$ & $31(19,39)$ & $10(8.0,14)$ \\
\hline \multirow[t]{2}{*}{ MND } & $10(6.6,16)$ & $130(86,230)$ & $13(10,17)$ & $27(16,39)$ & $11(4.9,18)$ \\
\hline & poly-GR & & & & \\
\hline FTLD & $3.0(2.2,4.2)$ & $16(9.5,48)$ & $3.0(2.3,5.6)$ & $5.1(3.3,9.3)$ & $2.6(1.3,5.5)$ \\
\hline FTLD-MND & $6.8(3.7,9.5)^{*}$ & $49(26,86)^{*}$ & $8.5(6.8,12)^{*}$ & $17(11,26)^{*}$ & $4.7(3.1,7.9)$ \\
\hline \multirow[t]{2}{*}{ MND } & $2.9(1.1,3.4)$ & $26(18,51)$ & $7.5(4.7,8.8)$ & $12(3.7,22)$ & $2.6(1.2,4.9)$ \\
\hline & aDMA & & & & \\
\hline FTLD & $3.3(2.8,4.7)$ & $83(31,100)$ & $10(6.8,14)$ & $16(10,37)$ & $3.4(2.1,5.6)$ \\
\hline FTLD-MND & $5.5(3.1,8.2)$ & $77(21,140)$ & $17(14,22)^{*}$ & $25(17,36)$ & $5.6(4.6,6.8)$ \\
\hline MND & $4.0(2.1,7.4)$ & $53(31,81)$ & $15(12,20)$ & $15(6,29)$ & $3.5(2.5,5.6)$ \\
\hline
\end{tabular}

In frontal cortex, $p<0.001$, FTLD vs. FTLD-MND, $p=0.005$, FTLD-MND v.s MND. In DF, $p=0.02$, FTLD vs. FTLD-MND. In CA4, $p=0.002$, FTLD vs. FTLD-MND. In CA2/3, $p=0.003$, FTLD vs. FTLD-MND. All variables analyzed with Kruskal-Wallis ANOVA on Ranks and data are displayed as median (25th and 75th range)

"Statistically significant $p$-value $(p<0.05)$; all $p$-value for ANOVA on Ranks comparison of all three groups

FCtx frontal cortex, MCtx Motor cortex, DF dentate fascia, CA (cornu ammonis)

cytoplasmic aggregates of GFP-(GR) ${ }_{100}$ cells. Treating cells with AdOx decreased cytoplasmic aggregates in GFP-(GR) 100 cells, while having no effect on the number of cells expressing GFP (Fig. $5 \mathrm{c}$ and d). To confirm immunofluorescent findings, we performed western blot analysis. Consistent with the results of immunostaining, only GFP-(GR) $)_{100}$ cells showed evidence of high molecular weight aDMA-immunoreactive species (arrows in Fig. 6). These results suggest that poly-GR aggregation may be modulated by arginine methylation.

\section{Discussion}

An expanded hexanucleotide repeat in C9ORF72 is the most frequent cause of FTLD and MND. One consequence of the expanded repeats is formation of DPR polymers through unconventional RAN translation [1, 21]. Despite increasing evidence from animal and cell culture models suggesting individual DPR have different degrees of toxicity, the manifestations of this toxicity and pathomechanisms are poorly understood in the human brain. Moreover, the relationship of DPR-related neuropathology with various clinicopathologic subtypes of C9ORF72-related disease is not well understood. In this study, we focused on poly-GR and provide evidence that density of poly-GR inclusions correlates with neurodegeneration more robustly than other DPR, especially in individuals with the most severe clinicopathologic phenotype, FTLD-MND. In addition, we explored a possible association of poly-GR toxicity with post-translational modification by methylation. In clinicopathological analysis, we demonstrated differences in distribution of poly-GR inclusions compared with poly-GA and found that certain brain regions, such as the hippocampal DF, have abundant poly-GA inclusions, but very few poly-GR inclusions. Moreover, poly-GR inclusions are closely associated with histologic features of neurodegeneration, especially in the hippocampus and FCtx. We found a similar distribution of poly-GR and aDMA-positive inclusions, and co-localization of poly-GR with aDMA. Of the three clinicopathologic phenotypes of C9ORF72-related disease, the FTLD-MND subgroup had the most inclusions immunoreactive for poly-GR and aDMA, especially in the FCtx and in several subregions of the hippocampus.

Only a few studies have addressed possible differences in DPR species in clinicopathological subtypes of C9ORF72-related disease. A semiquantitative neuropathologic study by Mackenzie and coworkers of 8 cases of FTLD, 16 of FTLD-MND and 11 of MND, found no relationship between specific DPR species and clinicopathologic phenotype, except for moderate association of poly-GA-positive dystrophic neurites with histologic markers of degeneration in the frontal cortex [17]. In another semiquantitative study by Schludi and coworkers in a cohort of 3 FTLD, 4 FTLD-MND and 2 MND, poly-PR inclusions were more abundant in FTLD than MND in the hippocampus, while poly-GA inclusions were more abundant in cerebellar granular layer of 


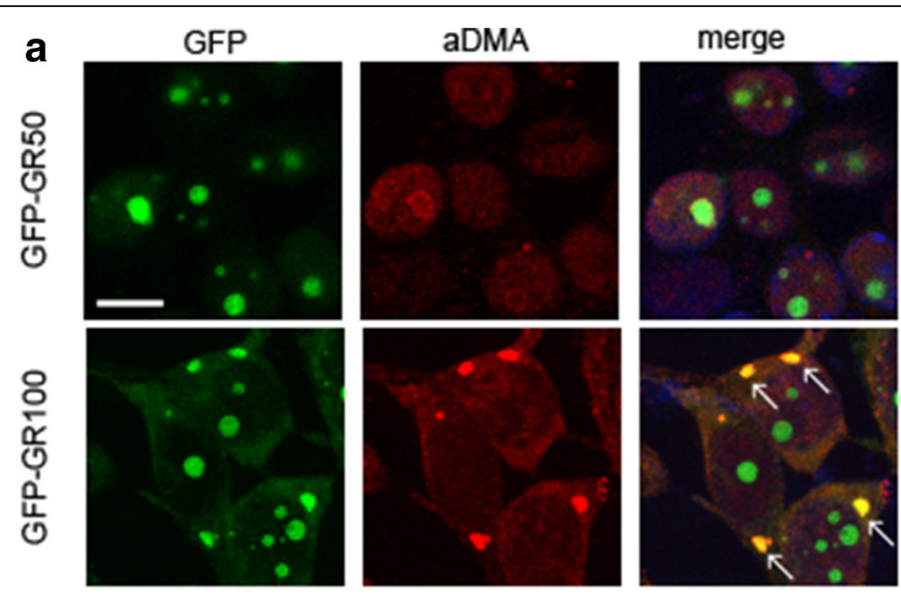

b
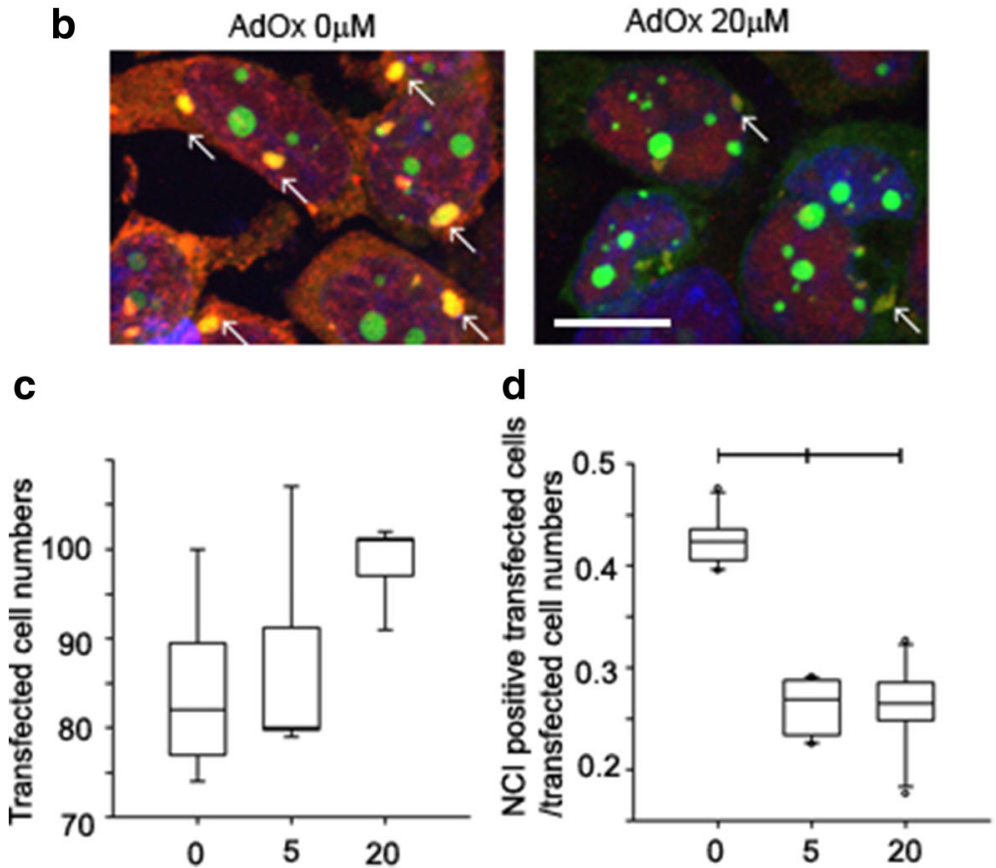

Fig. 5 Poly-GR proteins form cytoplasmic inclusions in HEK-293 T cultured cells. a Expression of GFP-(GR) $)_{50}$ in HEK-293 T cells accumulate in nucleus. No cytoplasmic inclusions are detected. Expression of GFP-(GR) 100 in HEK-293 T cells show immunoreactivity in nucleus and cytoplasm, with formation of cytoplasmic inclusions. Double immunofluorescence of GFP-(GR) $)_{100}$ and aDMA in HEK-293 T cells. b GFP-(GR) 100 and aDMA. GFP-(GR) 100 and no arginine methyltransferase inhibitor (AdOx) (left) versus AdOx $20 \mu \mathrm{M}$ (right). Arrows indicate poly-GR and aDMA positive cytoplasmic aggregation (c, d) Effects of AdOx on poly-GR100 cytoplasmic inclusion formation. AdOx did not significantly decrease GFP positive, transfected cell numbers. c AdOx significantly decreased ratio of cytoplasmic inclusions in transfected cells $-43 \%$ at $0 \mathrm{mM}, 27 \%$ at $5 \mathrm{mM}$ and $25 \%$ at $20 \mathrm{mM}$. d Quantitative analysis and representative image showing cytoplasmic inclusions at different concentration of AdOx. Counts were made of over 500 transfected cells for each condition (with at least duplicate counts performed for each field analyzed). All variables analyzed with Kruskal-Wallis ANOVA on Ranks followed by Tukey's post hoc analysis and data are displayed as median (25th and 75th range). *Statistically significant $p$-value $(p<0.05)$; all $p$-values for ANOVA on Ranks comparison of all three groups. Scale bars in (a) and (b): $20 \mu \mathrm{m}$

FTLD than both FTLD-MND and MND cases, but neither poly-PR nor poly-GA correlated with neurodegeneration [26]. In contrast, we found correlations between poly-GR and neurodegeneration in a larger series of cases using quantitative digital microscopic methods. Another unique finding in our study was that patients with the most severe clinicopathologic phenotype, namely those with both FTLD and MND, had higher lesion burden that the other two clinicopathologic groups. Like Schludi and coworkers [26], we found the highest density of poly-GR in FTLD-MND. On the other hand, we were not able to detect any difference between FTD and MND. The FTD group had the oldest age at death and longest disease duration; therefore, greater neurodegeneration might be associated with clearance of poly-GR pathology. While the three largest neuropathologic studies 


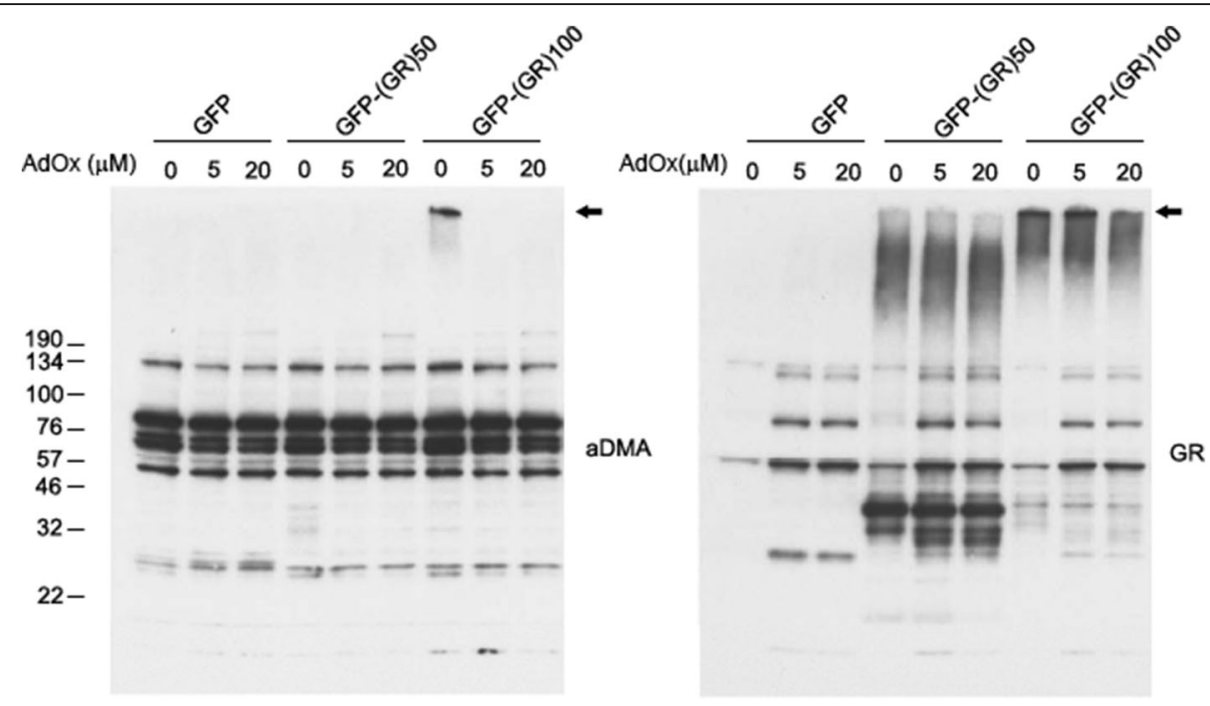

GFP

Fig. 6 Western blot analysis of soluble and insoluble cell lysates. Immunoblots show poly-GR in high molecular weight species. Arrows mark the top of the gel. Only GFP-(GR) 100 cells had dimethylarginine in high molecular species, which was lost after treatment with AdOx at 5- $\mu M$ and $20-\mu \mathrm{M}$. ${ }^{*} p<0.05$. ANOVA All $p$-value for ANOVA on ranks comparison of all three groups. Mann-Whitney $U$ test was employed for the statistical analysis

of DPR pathology in C9ORF72-related disease and a range of clinicopathologic subtypes, different quantitative methods and different antibodies for immunohistochemistry, there were several notable similarities. All found that poly-GR inclusions were less abundant than poly-GA (and poly-GP) inclusions and that certain brain regions had propensity for DPR inclusions, such as specific hippocampal subregions (DF, CA4 and CA2/3) and the cerebellar internal granular cell layer. The current study is the first to use digital pathology and quantitative methods, including color deconvolution image analysis, to objectively assess the burden of DPR pathology and histologic markers of neurodegeneration.

A recent study by Saberi and coworkers suggested poly-GR inclusions co-localize with TDP-43 in dendritic structures in a small series $(n=5)$ of MND compared with sporadic ALS $(n=3)$ [25]. These findings differ from our results and those of others $[17,26]$, who find little evidence for poly-GR immunoreactive dystrophic neurites.

Various pathomechanisms have been proposed for C9ORF72-related disease based on animal and cell culture models, including loss of function of C9ORF72 leading to neuroinflammation [23], nuclear dysfunction due to alternative RNA splicing, perturbations in RNA metabolism, formation of DNA/RNA hybrids, RNA foci and defects in nucleocytoplasmic transport. The most characteristic pathologic hallmark of C9ORF72-related is widespread and regionally-specific neuronal DPR polymer inclusions and RNA foci. Given that two of the DPR contain arginine polymers, we hypothesized that post-translational modification of these DPR by methylarginine transferases might uniquely contribute to their greater toxicity. We found evidence to support methylation of poly-GR, while poly-PR was not amenable to 
study due to the paucity of these inclusions and the lack of reliable and sensitive reagents for their detection. Double immunostaining methods showed colocalization of aDMA immunoreactivity in poly-GR inclusions (Fig. 3). In contrast, there was very little colocalization of poly-GA and aDMA in neuronal inclusions (data not shown). Moreover, the distribution of poly-GR was highly correlated with the distribution of aDMA (Additional file 6: Table S2). We found that aDMA immunoreactivity was present in normal nuclei of various cell types, which is assumed to be physiologic methylation of nuclear proteins, such as histones and RNA-binding proteins [10]. Boeynaems and coworkers previously reported nuclear signal of aDMA in dentate fascia neurons of healthy controls [3]. We also detected nuclear aDMA in control cases (Additional file 7: Figure S5). Therefore, it is reasonable the aDMA nuclear labeling represents physiological methylation. In these proteins, DMA posttranslational modification occurs at sites with a consensus glycine/arginine rich (GAR) motif, which may be mimicked by poly-GR. Recent studies show that in addition to GAR-domain class of proteins, other proteins can be targeted by arginine methyltransferases [10]. Based upon histologic features and morphology, the nuclear aDMA we detected was not only in intranuclear inclusions, but also in other nuclear structures. Unlike normal controls, in c9FTLD-MND aDMA was found in the neuronal cytoplasm and it co-localized with poly-GR, especially in the hippocampus.

Given the strong correlation between poly-GR and aDMA, their association with neurodegeneration, and their relative abundance in patients with the most severe clinicopathologic phenotype (FTLD-MND), we hypothesized that post-translational modification of poly-GR may be a novel mechanism of gain of toxicity of poly-GR. In support of this, poly-GR cytoplasmic inclusions in cultured cells expressing GFP-(GR) 100 were frequently co-labeled with a sensitive and specific antibody to aDMA. Treating the cells with an arginine methyltransferase inhibitor (AdOx), which has intrinsic cytotoxic properties, decreased the numbers of poly-GR cytoplasmic inclusions at times and concentrations not associated with significant cytotoxicity. These findings suggest that formation of poly-GR cytoplasmic inclusions might be influenced by aDMA modification.

Although post-translational modification of proteins with aDMA is often toxic in various tissues in human diseases, including the brain [5, 27, 29], the effects of post-translational aDMA modification is not well understood in the brain. Regarding clinical and experimental studies of CNS disease and aDMA, in hepatic encephalopathy, elevated aDMA levels could potentially contribute to cognitive dysfunction through oxidative stress, restriction of cerebral blood flow and inflammation [5].
In ischemic stroke, elevated levels of aDMA might contribute to brain injury through endothelial cell damage, and aDMA also might be involved in nitric oxide associated oxidative stress and excitotoxicity [4]. In neurodegenerative disorders, Suarez-Calved and coworkers reported that mono-methylated arginine is frequent in FTLD-FUS, but not in ALS-FUS. Unmethylated and mono-methylated methylarginine FUS had much higher binding affinities to the nuclear receptor of FUS, transportin-1, compared to aDMA FUS [28]. To complicate matters, DMA modification is also considered to both positively and negatively regulate protein-protein interactions [10].

A limitation of our study is the inability to detect specific methylated forms (e.g., mono-methylation, symmetrical di-methylation and asymmetrical di-methylation) of poly-GR (and poly-PR). There is also no direct way to know the effect of the DPR polymer repeat length on post-translational modification. Our in vitro cell culture studies used repeat lengths of 50- and 100-mers. These are much smaller repeats that seen in disease brain based upon their mobility on western blots. In this regard, it is interesting to note that Bennion Callister and co-workers pointed out that repeat length effects subcellular distribution of DPR, with smaller repeats producing inclusions in the nucleus (specifically in nucleoli) and larger repeats $(>1000)$ producing inclusions in the cytoplasm [2]. We were able to detect both nuclear and cytoplasmic inclusions in our cell culture models with much smaller repeats. It remains to be determined if repeat length affects methylation modification. Nevertheless, we suggest that current studies provide preliminary evidence from human neuropathology that post-translational modification of arginine residues may play a role in the toxicity of poly-GR. Clearly, there is need for further study.

\section{Conclusions}

In summary, we provide evidence that poly-GR is more closely associated with markers of neurodegeneration than poly-GA and poly-GP. Preliminary evidence suggests that methylation may contribute to this toxicity. Furthermore, in our small clinicopathologic series poly-GR seems to be more abundant in patients with FTLD-MND compared to FTLD or MND. Investigating the contribution of poly-GR to neuronal dysfunction, TDP-43 pathology and neurodegeneration may shed additional light on disease pathogenesis and lead to new therapeutic targets.

\section{Additional files}

Additional file 1: Figure S1. Semiquantitative assessment of neurodegeneration. Grading neurodegeneration in FCtx, hippocampal DF, CA4 and CA2/3 are shown. In FCtx, neurodegeneration was assessed by superficial microvacuolation and spongiosis. In DF, gaps in granular cell density were assessed as neurodegeneration. In CA4 and CA2/3, 
neuronal cell loss and gliosis were assessed as neurodegeneration. Neurodegeneration was graded as absent (G0), mild (G1), moderate (G2) or severe (G3). (TIF $1630 \mathrm{~kb}$ )

Additional file 2: Figure S2. Correlation between manual counts and positive pixel burden from color deconvolution in poly-GR staining. Plot shows the correlation of manual counts of neuronal cytoplasmic inclusions and positive pixel burden from color deconvolution in poly-GR staining. The line shows linear regression CD color deconvolution. Ctx frontal cortex, DF dentate fascia, CA - cornu ammonis, MCtx motor cortex. (TIF $2581 \mathrm{~kb}$ )

Additional file 3: Table S1. Quantitative assessment of DPR density by color deconvolution algorithm in clinicopathologic subgroups of C9ORF72-related disease. In frontal cortex, $p=0.019$, FTLD vs. FTLD-MND. In CA4, $p=0.055$, FTLD vs. FTLD-MND. In CA2/3, $p=0.03$, FTLD vs. FTLDMND. Significant $p$-values $(<0.05)$ are indicated in bold. All variables were analyzed with Kruskal-Wallis ANOVA on Ranks and data are displayed as median (25th and 75th range). *Statistically significant $p$-value $(p<0.05)$; all p-value for ANOVA on Ranks comparison of all three groups. FCtx = frontal cortex, MCtx = Motor cortex, DF dentate fascia, CA hippocampal subfields. (DOCX $15 \mathrm{~kb}$ )

Additional file 4: Figure S3. Dot plot graph of semiquantitative assessment of neurodegeneration and DPR. Note that $X$ axis is neurodegeneration score (0 to 3), Y-axis is density of DPR. FCtx - frontal cortex, DF - dentate fascia, CA4 - cornu ammonis sector 4. (TIF $1325 \mathrm{~kb}$ )

Additional file 5: Figure S4. Western blot analysis of aDMA and SDMA in brains of C9FLTD-MND and sporadic FTLD-MND. The high molecular weight aDMA and SDMA signals are visible in C9FTD/ALS, but not in sporadic FTD/ ALS cases. (TIF $2960 \mathrm{~kb}$ )

Additional file 6: Table S2. Correlation between DPR and aDMA inclusions. $P$-values are from Pearson's test of correlation. Significant $p$-values $(<0.05)$ are indicated in bold. FCtx frontal cortex, DF dentate fascia, CA cornu ammonis, MCtx motor cortex. (DOCX $14 \mathrm{~kb}$ )

Additional file 7: Figure S5 Comparison of immunostaining with aDMA between C9ORF72 cases and non- neurodegeneration control in parahippocampal cortex. The nuclear signal of aDMA is variable in both cases and controls. Note sparse cytoplasmic inclusions labeled with aDMA in in C9ORF72 cases (arrows). Scale bar represents $50 \mu \mathrm{M}$. (TIF $2898 \mathrm{~kb}$ )

\section{Acknowledgements}

We thank the patients and their families who donated brains to help further our knowledge of C9FTLD-MND. We are grateful to Linda Rousseau and Virginia Phillips for histological support; and to Monica Castanedes-Casey for immunohistochemistry support.

\section{Funding}

This study is supported by NIH: P01 NS084974-04, R01 AG037491-08, P50 AG016574-19.

\section{Availability of data and materials}

The data and materials, including digital pathology and glass slides, are available for sharing upon request.

\section{Authors' contributions}

NS, KFB, Y-JZ, KR, TFG, MEM and RR contributed to data acquisition and analysis. LP and DWD provided support and scientific oversight for the studies. All authors contributed in writing the final manuscript. All authors have approved the final version of the manuscript.

\section{Ethics approval and consent to participate}

All autopsies were performed after informed consent of the legal-nextof-kin or someone with legal authority to grant permission. The brain bank for neurodegenerative disorders at Mayo Clinic operates under approved guidelines of the Mayo Clinic Institutional Review Board and studies on autopsy samples are considered exempt from human subjects research under 45 CFR 46.101.

\section{Consent for publication}

The study is performed as part of research funded by the National Institutes of Health and is intended to be widely available to the public.

\section{Competing interests}

The authors declare that they have no competing interests.

\section{Publisher's Note}

Springer Nature remains neutral with regard to jurisdictional claims in published maps and institutional affiliations.

Received: 26 June 2018 Accepted: 30 June 2018

Published online: 20 July 2018

\section{References}

1. Ash PE, Bieniek KF, Gendron TF, Caulfield T, Lin WL, Dejesus-Hernandez M, van Blitterswijk MM, Jansen-West K, Paul JW 3rd, Rademakers R, Boylan KB, Dickson DW, Petrucelli L (2013) Unconventional translation of C9ORF72 GGGGCC expansion generates insoluble polypeptides specific to C9FTD/ALS Neuron 77:639-646

2. Bennion Callister J, Ryan S, Sim J, Rollinson S, Pickering-Brown SM (2016) Modelling C9orf72 dipeptide repeat proteins of a physiologically relevant size. Hum Mol Genet 25:5069-5082

3. Boeynaems S, Bogaert E, Michiels E, Gijselinck I, Sieben A, Jovicic A, De Baets G, Scheveneels W, Steyaert J, Cuijt I, Verstrepen KJ, Callaerts P. Rousseau F, Schymkowitz J, Cruts M, Van Broeckhoven C, Van Damme P, Gitler AD, Robberecht W, Van Den Bosch L (2016) Drosophila screen connects nuclear transport genes to DPR pathology in C9ALS/FTD. Sci Rep 6:20877

4. Chen S, Li N, Deb-Chatterji M, Dong Q, Kielstein JT, Weissenborn K, Worthmann H (2012) Asymmetric dimethyarginine as marker and mediator in ischemic stroke. Int J Mol Sci 13:15983-16004

5. Czarnecka A, Milewski K, Zielinska M (2017) Asymmetric Dimethylarginine and hepatic encephalopathy: cause, effect or association? Neurochem Res 42:750-761

6. Davidson YS, Barker H, Robinson AC, Thompson JC, Harris J, Troakes C, Smith B, Al-Saraj S, Shaw C, Rollinson S, Masuda-Suzukake M, Hasegawa M, Pickering-Brown S, Snowden JS, Mann DM (2014) Brain distribution of dipeptide repeat proteins in frontotemporal lobar degeneration and motor neurone disease associated with expansions in C9ORF72. Acta Neuropathol Commun 2:70

7. DeJesus-Hernandez M, Mackenzie IR, Boeve BF, Boxer AL, Baker M, Rutherford NJ, Nicholson AM, Finch NA, Flynn H, Adamson J, Kouri N, Wojtas A, Sengdy P, Hsiung GY, Karydas A, Seeley WW, Josephs KA, Coppola G, Geschwind DH, Wszolek ZK, Feldman H, Knopman DS, Petersen RC, Miller BL, Dickson DW, Boylan KB, Graff-Radford NR, Rademakers R (2011) Expanded GGGGCC hexanucleotide repeat in noncoding region of C9ORF72 causes chromosome 9p-linked FTD and ALS. Neuron 72:245-256

8. Dickson DW, Josephs KA, Amador-Ortiz C (2007) TDP-43 in differential diagnosis of motor neuron disorders. Acta Neuropathol 114:71-79

9. Freibaum BD, Taylor JP (2017) The role of dipeptide repeats in C9ORF72related ALS-FTD. Front Mol Neurosci 10:35

10. Gayatri S, Bedford MT (2014) Readers of histone methylarginine marks. Biochim Biophys Acta 1839:702-710

11. Gendron TF, Bieniek KF, Zhang YJ, Jansen-West K, Ash PE, Caulfield T, Daughrity L, Dunmore JH, Castanedes-Casey M, Chew J, Cosio DM, van Blitterswijk M, Lee WC, Rademakers R, Boylan KB, Dickson DW, Petrucelli L (2013) Antisense transcripts of the expanded C9ORF72 hexanucleotide repeat form nuclear RNA foci and undergo repeat-associated non-ATG translation in C9FTD/ALS. Acta Neuropathol 126:829-844

12. Geser F, Brandmeir NJ, Kwong LK, Martinez-Lage M, Elman L, McCluskey L, Xie SX, Lee VM, Trojanowski JQ (2008) Evidence of multisystem disorder in whole-brain map of pathological TDP-43 in amyotrophic lateral sclerosis. Arch Neurol 65:636-641

13. Geser F, Stein B, Partain M, Elman LB, McCluskey LF, Xie SX, Van Deerlin VM, Kwong LK, Lee VM, Trojanowski JQ (2011) Motor neuron disease clinically limited to the lower motor neuron is a diffuse TDP-43 proteinopathy. Acta Neuropathol 121:509-517

14. Josephs KA, Stroh A, Dugger B, Dickson DW (2009) Evaluation of subcortical pathology and clinical correlations in FTLD-U subtypes. Acta Neuropathol 118:349-358

15. Kwon I, Xiang S, Kato M, Wu L, Theodoropoulos P, Wang T, Kim J, Yun J, Xie Y. McKnight SL (2014) Poly-dipeptides encoded by the C9orf72 repeats bind nucleoli, impede RNA biogenesis, and kill cells. Science 345:1139-1145 
16. Mackenzie IR, Arzberger T, Kremmer E, Troost D, Lorenzl S, Mori K, Weng SM, Haass C, Kretzschmar HA, Edbauer D, Neumann M (2013) Dipeptide repeat protein pathology in C9ORF72 mutation cases: clinico-pathological correlations. Acta Neuropathol 126:859-879

17. Mackenzie IR, Frick P, Grasser FA, Gendron TF, Petrucelli L, Cashman NR, Edbaue D, Kremmer E, Prudlo J, Troost D, Neumann M (2015) Quantitative analysis and clinico-pathological correlations of different dipeptide repeat protein pathologies in C9ORF72 mutation carriers. Acta Neuropathol 130:845-861

18. Mackenzie IR, Neumann M, Baborie A, Sampathu DM, Du Plessis D, Jaros E, Perry RH, Trojanowski JQ, Mann DM, Lee VM (2011) A harmonized classification system for FTLD-TDP pathology. Acta Neuropathol 122:111-113

19. Mann DM (2015) Dipeptide repeat protein toxicity in frontotemporal lobar degeneration and in motor neurone disease associated with expansions in C9ORF72-a cautionary note. Neurobiol Aging 36:1224-1226

20. Mizielinska S, Gronke S, Niccoli T, Ridler CE, Clayton EL, Devoy A, Moens T, Norona FE, Woollacott IOC, Pietrzyk J, Cleverley K, Nicoll AJ, Pickering-Brown S, Dols J, Cabecinha M, Hendrich O, Fratta P, Fisher EMC, Partridge L, Isaacs AM (2014) C9orf72 repeat expansions cause neurodegeneration in Drosophila through arginine-rich proteins. Science 345:1192-1194

21. Mori K, Arzberger T, Grasser FA, Gijselinck I, May S, Rentzsch K, Weng SM, Schludi MH, van der Zee J, Cruts M, Van Broeckhoven C, Kremmer E, Kretzschmar HA, Haass C, Edbauer D (2013) Bidirectional transcripts of the expanded C9orf72 hexanucleotide repeat are translated into aggregating dipeptide repeat proteins. Acta Neuropathol 126:881-893

22. Murray ME, Przybelski SA, Lesnick TG, Liesinger AM, Spychalla A, Zhang B, Gunter JL, Parisi JE, Boeve BF, Knopman DS, Petersen RC, Jack CR Jr, Dickson DW, Kantarci K (2014) Early Alzheimer's disease neuropathology detected by proton MR spectroscopy. J Neurosci 34:16247-16255

23. O'Rourke JG, Bogdanik L, Yanez A, Lall D, Wolf AJ, Muhammad AK, Ho R, Carmona S, Vit JP, Zarrow J, Kim KJ, Bell S, Harms MB, Miller TM, Dangler CA, Underhill DM, Goodridge HS, Lutz CM, Baloh RH (2016) C9orf72 is required for proper macrophage and microglial function in mice. Science 351:1324-1329

24. Renton AE, Majounie E, Waite A, Simon-Sanchez J, Rollinson S, Gibbs JR, Schymick JC, Laaksovirta H, van Swieten JC, Myllykangas L, Kalimo H, Paetau A, Abramzon Y, Remes AM, Kaganovich A, Scholz SW, Duckworth J, Ding J, Harmer DW, Hernandez DG, Johnson JO, Mok K, Ryten M, Trabzuni D, Guerreiro RJ, Orrell RW, Neal J, Murray A, Pearson J, Jansen IE, Sondervan D, Seelaar $H$, Blake D, Young K, Halliwell N, Callister JB, Toulson G, Richardson A, Gerhard A, Snowden J, Mann D, Neary $D$, Nalls MA, Peuralinna $T$, Jansson $L$, Isoviita VM, Kaivorinne $A L$, Holtta-Vuori M, Ikonen E, Sulkava R, Benatar M, Wuu J, Chio A, Restagno G, Borghero G, Sabatelli M, Heckerman D, Rogaeva E, Zinman L, Rothstein JD, Sendtner M, Drepper C, Eichler EE, Alkan C, Abdullaev Z, Pack SD, Dutra A, Pak E, Hardy J, Singleton A, Williams NM, Heutink P, Pickering-Brown S, Morris HR, Tienari PJ, Traynor BJ (2011) A hexanucleotide repeat expansion in C9ORF72 is the cause of chromosome 9p21-linked ALS-FTD. Neuron 72:257-268

25. Saberi S, Stauffer JE, Jiang J, Garcia SD, Taylor AE, Schulte D, Ohkubo T, Schloffman CL, Maldonado M, Baughn M, Rodriguez MJ, Pizzo D, Cleveland D, Ravits J (2017) Sense-encoded poly-GR dipeptide repeat proteins correlate to neurodegeneration and uniquely co-localize with TDP-43 in dendrites of repeat-expanded C9orf72 amyotrophic lateral sclerosis. Acta Neuropathol

26. Schludi MH, May S, Grasser FA, Rentzsch K, Kremmer E, Kupper C, Klopstock T, Arzberger T, Edbauer D (2015) Distribution of dipeptide repeat proteins in cellular models and C9orf72 mutation cases suggests link to transcriptional silencing. Acta Neuropathol 130:537-555

27. Schwedhelm E, Boger RH (2011) The role of asymmetric and symmetric dimethylarginines in renal disease. Nat Rev Nephrol 7:275-285

28. Suarez-Calvet $M$, Neumann $M$, Arzberger $T$, Abou-Ajram C, Funk $E_{\text {, }}$ Hartmann H, Edbauer D, Kremmer E, Gobl C, Resch M, Bourgeois B, Madl T, Reber S, Jutzi D, Ruepp MD, Mackenzie IR, Ansorge O, Dormann D, Haass C (2016) Monomethylated and unmethylated FUS exhibit increased binding to Transportin and distinguish FTLD-FUS from ALS-FUS. Acta Neuropathol 131:587-604

29. Tain YL, Hsu CN (2017) Toxic Dimethylarginines: asymmetric Dimethylarginine (ADMA) and symmetric Dimethylarginine (SDMA). Toxins (Basel) 9

30. Tao Z, Wang H, Xia Q, Li K, Li K, Jiang X, Xu G, Wang G, Ying Z (2015) Nucleolar stress and impaired stress granule formation contribute to C9orf72 RAN translation-induced cytotoxicity. Hum Mol Genet 24:2426-2441
31. Todd TW, Petrucelli $L$ (2016) Insights into the pathogenic mechanisms of chromosome 9 open reading frame 72 (C9orf72) repeat expansions. J Neurochem 138(Suppl 1):145-162

32. Vatsavayai SC, Yoon SJ, Gardner RC, Gendron TF, Vargas JN, Trujillo A, Pribadi M, Phillips JJ, Gaus SE, Hixson JD, Garcia PA, Rabinovici GD, Coppola G, Geschwind DH, Petrucelli L, Miller BL, Seeley WW (2016) Timing and significance of pathological features in C9orf72 expansion-associated frontotemporal dementia. Brain 139:3202-3216

33. Wen X, Tan W, Westergard T, Krishnamurthy K, Markandaiah SS, Shi Y, Lin S, Shneider NA, Monaghan J, Pandey UB, Pasinelli P, Ichida JK, Trotti D (2014) Antisense proline-arginine RAN dipeptides linked to C9ORF72-ALS/FTD form toxic nuclear aggregates that initiate in vitro and in vivo neuronal death. Neuron 84:1213-1225

34. Yang D, Abdallah A, Li Z, Lu Y, Almeida S, Gao FB (2015) FTD/ALS-associated poly(GR) protein impairs the notch pathway and is recruited by poly(GA) into cytoplasmic inclusions. Acta Neuropathol 130:525-535

\section{Ready to submit your research? Choose BMC and benefit from:}

- fast, convenient online submission

- thorough peer review by experienced researchers in your field

- rapid publication on acceptance

- support for research data, including large and complex data types

- gold Open Access which fosters wider collaboration and increased citations

- maximum visibility for your research: over $100 \mathrm{M}$ website views per year

At BMC, research is always in progress.

Learn more biomedcentral.com/submissions 